\title{
An Opportunity for Cognitive Task Analysis in Neonatal Resuscitation
}

\author{
Emily C. Zehnder ${ }^{1,2}$, Brenda H. Y. Law ${ }^{1,2}$ and Georg M. Schmölzer ${ }^{1,2 *}$ \\ ${ }^{1}$ Neonatal Research Unit, Centre for the Studies of Asphyxia and Resuscitation, Royal Alexandra Hospital, Edmonton, AB, \\ Canada, ${ }^{2}$ Department of Pediatrics, University of Alberta, Edmonton, AB, Canada
}

Approximately $10 \%$ of newborn infants require resuscitative intervention at birth. Ideally, this care is provided by a team of expert healthcare professionals who possess exceptional cognitive, psychomotor, and communication skills. Human errors and deviations from resuscitation protocol are common and may be attributable to excessive cognitive demand experienced by the resuscitation team. Cognitive Task Analysis (CTA) is a group of methods used to assess knowledge, judgments, goals, and decisionmaking of expert healthcare professionals. These methods may be used during neonatal resuscitation to gain an improved understanding of the approaches used by healthcare professionals. CTA methods have been applied in many medical disciplines including neonatology. CTA has been used to identify information previously confined to the intuition of experts. This information has been used to assess, develop, and improve medical technology, clinical decision support tools (DSTs), communication structure, and training methods. Knowledge attained through CTA might be applied similarly to neonatal resuscitation, which may in turn decrease human errors, and improve patient safety.

Anup C. Katheria, Sharp Mary Birch Hospital for Women \& Newborns, United States

Reviewed by:

Maria Den Boer,

Leiden University Medical

Center, Netherlands

Maria Fernanda Almeida,

Federal University of São Paulo, Brazil

*Correspondence:

Georg M. Schmölzer

georg.schmoelzer@me.com

Specialty section:

This article was submitted to Neonatology,

a section of the journal

Frontiers in Pediatrics

Received: 21 May 2019 Accepted: 14 August 2019 Published: 27 August 2019

Citation:

Zehnder EC, Law BHY and Schmölzer GM (2019) An Opportunity for Cognitive Task Analysis in Neonatal

Resuscitation. Front. Pediatr. 7:356. doi: 10.3389/fped.2019.00356
Keywords: neonatal resuscitation, delivery room, cognitive task analysis, knowledge elicitation, decision support tools

\section{INTRODUCTION}

During neonatal resuscitation, decisions must be made quickly and healthcare professionals (HCPs) must possess exceptional cognitive, psychomotor, and communication skills to identify problems, analyze complex scenarios, generate solutions, and refine a large amount of data into useful information under time pressure (1). This situation often leads to human errors and deviations from resuscitation protocols (2). In addition, two-thirds of preventable infant mortality/sentinel events during neonatal resuscitation are caused by poor communication or breakdown of teamwork (3-5). Therefore, understanding the cognitive, psychomotor, and communication processes used by expert HCPs might identify factors associated with human error.

Cognitive Task Analysis (CTA) can be used to examine cognitive, psychomotor, and communication processes, and to provide solutions to improve HCPs communication and education. CTA is a mechanism for capturing expertise; by breaking down complex cognitive processes that drive a set of behaviors, one can formulate comprehensive algorithmic descriptions of tasks and define task rules $(6,7)$. CTA is a diverse group of methods; more than 100 different systematic and scientific CTA have been described (8). For example, The Think Aloud Method is a simple and relatively well-known method of CTA. During a Think Aloud a subject matter expert (SME) verbalizes their thought process while completing a task (9). The Critical Decision Method (CDM) is another frequently used method of CTA; this method involves a retrospective interview in which participants are asked to recall a non-routine incident and are probed about decisions and judgments during that incident (10). While specific elements of each CTA approach 
differ, five steps are common: (1) collection of preliminary knowledge, (2) identification of knowledge representations, (3) application of focused knowledge elicitation methods, (4) analysis and verification of data acquired, and (5) formulation of results for intended applications (7). This review aims to provide an overview of previous applications of CTA in neonatology and related medical disciplines and to identify how CTA could be applied to neonatal resuscitation.

\section{MEDICAL EQUIPMENT}

During neonatal resuscitation, equipment must be reliable, userfriendly, and allow for easy detection of equipment flaws. Malfunctioning of equipment or user-interface issues could result in increased stress and cognitive load in HCPs, which might result in detrimental effects on patient outcomes. CTA could be used to assess how HCPs interact with medical technology, and how they recognize and cope with equipment failure.

CTA has been used to assess vital sign monitors, and incubators $(11,12)$. Li et al. assessed how neonatal HCPs retrieve data on vital sign monitors and identified several challenges including overwhelming alarm noises and difficulties assessing unusual events (11). This led to the development of automated aids for cardio-respiratory trend retrieval and alarm limits adjustment (11). Similarly, Ferris et al. developed blueprints of incubator systems based on CTA assessment that would better fit the needs of infants, parents, and HCPs (12). CTA identified several flaws including confusing alarm information, ambiguous commands with scale functions, unintuitive icon displays, and lack of variability in alarms with existing incubator systems, and provided solutions (12). These examples demonstrate that CTA could be used to improve neonatal resuscitation equipment for HCP use.

\section{CLINICAL DECISION SUPPORT TOOLS}

Decision support tools (DSTs) are designed to decrease the cognitive load of HCPs, improve quality of care, and decrease human errors by linking health observations with health knowledge. Though many DSTs are electronic, any tools, designs or systems which links health observations to health knowledge maybe considered a DST. DSTs cover a portion of the cognitive load previously allocated to a HCP, freeing up cognitive resources for other tasks (13). This is important as excess cognitive load has been associated with human error (14). DSTs have been developed for use in neonatal resuscitation including visual and auditory reminders to prompt interventions. Fuerch et al. assessed the impact of a visual and auditory DST and reported significantly improved mask ventilation performance (94-95\% vs. $55-80 \%$ in intervention vs. control group, $p<0.0001$ ) and chest compressions ( $82-93 \%$ vs. $71-81 \%$ in intervention

Abbreviations: ACTA, Applied Cognitive Task Analysis; CDM, Critical decision method; DST, Decision Support Tool; ECPR, Pediatric Extracorporeal Cardiopulmonary Resuscitation; FLORENCE, Fuzzy LOgic REspiratory Neonatal Care Expert; HCP, Health care professionals; NICU, Neonatal Intensive Care Unit; SME, Subject matter experts. vs. control group, $p<0.0001)$ during simulated neonatal resuscitations (15).

CTA has been used in the design, optimization, and integration of various clinical DSTs (16-18). Schnittker et al. used CTA to aid in the design of a DST for use during challenging airway management in adult anesthesia (16). CDM interviews and focus groups with HCPs identified that the location of airway equipment was the main contributor to successful airway management $(16,17)$. Based on this assessment, Schnittker et al. customized an airway equipment trolley with a strategic layout and set-up to support decision making in anesthesia (17). This approach was decided on as anesthesia team members are thought to act through a recognition-primed process that links cues and actions, the equipment was positioned as to make these cues more salient to aid in recognition and decision making $(16,17)$.

Despite the benefits of DSTs, the acceptance rate is often low (19). There are several barriers preventing routine use of DSTs including perceived clinical irrelevance and discordance between cognitive processes and user interface (19). CTA may be used to overcome these barriers. One example is the development of Fuzzy LOgic REspiratory Neonatal Care Expert (FLORENCE). FLORENCE is a DST for managing ventilator settings in infants with respiratory distress syndrome (18). To ensure successful acceptance and integration of FLORENCE into clinical practice, three CTA based knowledge elicitation methods-Content Analysis, CDM, and observation-were used (18). Similarly, CTA methods including Cognitive Walkthrough and Think Aloud were used to assess the interaction of neonatal HCPs with an antibiotic-prescribing support tool (20). Cognitive Walkthrough aims to simulate a user's thought process as they interact with an interface (21). These methods identified several human computer interface problems, including lack of screen cues and ambiguous icons, which were associated with excessive cognitive efforts (20). These CTA approaches could also be used to optimize the design of DST for neonatal resuscitation.

\section{TEAMWORK AND COMMUNICATION}

During resuscitation, HCPs are reliant on team communication and a shared mental model, which serves as an exchange of patient information, education, coordination, and quality assurance. Ineffective communication can result in wasted resources, frustration, and errors, putting patients at risk (22). An improved understanding of cognitive processes involved in team communication during a neonatal resuscitation might guide the development of training and information systems to optimize team communication and performance. CTA can be used to better understand the dynamics of team communication. The benefits of CTA in analyzing team performance include understanding how teams interpret situations, make joint decisions, monitor team communication, and overcome confusion (6).

McHugh et al. used CDM in combination with direct observation to characterize communication among a multidisciplinary critical care team (23). McHugh et al. interviewed 
team members individually and identified barriers (e.g., fragmentary teams, role ambiguity, external collaborators, and novice difficulty in transitioning to from tactical to strategic) and facilitators (e.g., collaborative rounds, daily goal forms, and collaborative construction) to the development of a shared understanding and multi-disciplinary collaboration (23). Similarly, CTA-based interviews with HCPs in a pediatric intensive care unit identified the teams' cognitive framework in complex pediatric patient care (24). The interviews suggested that the care teams' efforts to create a shared mental model for their patients was central to the long-term care plan of patients across shift changes and hand-overs (24). Schraagen et al. used observation-based CTA to assess team performance in a pediatric cardiac surgery care team and compared them to surgical outcomes (25). A total of $255 \mathrm{~h}$ of operations were observed with a $76 \%$ inter-rater agreement and a $91 \%$ inter-rater reliability of the main four teamwork categories (25). In addition, CTA has been successfully used to coach effective teamwork through simulation design (26). These studies suggest that CTA might be an alternative approach to assess team performance and knowledge retention during neonatal resuscitations as well as an effective mechanism to optimize team behaviors.

\section{TRAINING}

When medical experts describe medical protocols or procedures to novice learners, they typically fail to describe $\sim 70 \%$ of the analytical and critical decisions required to successfully complete the task (27). These omissions are thought to be the experts' experiential knowledge, which is predominantly unconscious (27). CTA forces experts to consider all knowledge they use to complete a task, and in turn, make this information available to learners (28). In an assessment of education provided by surgical experts, CTA-prompted training resulted in an average of $22 \%$ more steps being described over unprompted teaching (28). By breaking down complex automated skills into bite-sized pieces, CTA could promote knowledge transfer from experts to non-experts.

\section{Benefits of CTA-Based Training}

CTA-based instruction has been associated with an average increase of $30-45 \%$ in learning (depending on CTA methods used) when compared to traditional task analysis methods (29). A meta-analysis of CTA-based instruction reported a large effect size (Hedges's $g=0.871$ ) (30). In addition, CTA-based education in surgery reported improved educational and surgical outcomes (e.g., including time, precision, and accuracy) and fewer errors compared to traditional learning methods (31).

However, the evidence of CTA-based medical education to teach neonatal trainees is limited. Crandall et al. used CDM interviews with expert Neonatal Intensive Care Unit (NICU) nurses to identify clinical symptoms for early sepsis detection (32). Overall, $36 \%$ of cues used by NICU nurses to correctly diagnose early sepsis were not reported in the medical literature or in training materials. These novel cues (e.g., "sick eyes," poor muscle tone, and edema) were subsequently added to training material and textbooks for future nursing students
(32). Similarly, CDM interviews with NICU nurses were used to identify indicators for necrotizing enterocolitis (e.g., context specific lethargy, color changes, and increased apneas) which were then shared with learners (33). CDM interviews with expert HCPs who perform neonatal resuscitation might reveal details previously overlooked or not thought of. This might be crucial information to improve learners' knowledge and improve outcomes for newborn infants.

\section{Expert-Novice Differences}

Expert-novice differences in clinical reasoning and decision making can be identified using CTA (34). Patterson et al. used CDM interviews to understand the differences between expert and novice HCPs in their recognition of sepsis in pediatric patients and reported that experts described more hypothesis testing and violated expectations compared to novices HCPs (34). These results were then used to develop an educational tool to train novice HCPs (34).

\section{Simulation-Based Training}

Simulations encourage experimental learning through the artificial representation of real scenarios to allow theoretical knowledge to be translated into clinical skills. This method does not put patients at risk and is independent of case availability. The benefits of simulation training in neonatal resuscitation include enhanced technical, behavioral and cognitive skill as well as improved team performance, and self-confidence (35). Regardless of learning method, skills are lost over time, which might be countered with periodic simulation training (36).

CTA could be used in simulation design to develop training scenarios and performance metrics. CTA could also help identify training needs and simulator requirements, which might result in a higher mental representation of leaners during simulation (37, 38). Cannon-Bowers et al. developed a five-step guide for simulation design using CTA. This method involves a Think Aloud followed by more focused probing to elicit critical cues, simulation deficiencies, and common errors (37). Munro et al. used a multistage CTA, called Concepts, Processes, and Principles, to elicit medical, instructional, and software expertise and integrate this into simulation design (39). During Concepts, Processes and Principles, SMEs are asked to create a gold standard list of steps involved in the completion of a task then describe critical concepts, processes, or principles that needs to be learned to explain and perform each step (29).

More recently, Pfandler et al. incorporated CTA into the design of team-based simulations. Pfandler et al. first defined all steps of the simulated procedure, then identified intra-operative technical and non-technical skills required by all involved professionals, and finally analyzed results (40). Patterson et al. used another approach involving CTA during the content and structure design of the simulations, which were then storyboarded (41). The resultant simulation was perceived as relevant and useful by learners ( $>70 \%$ of learners scored usefulness of simulation as $>7 / 10)(41)$.

Applied CTA (ACTA) has been used to assess the impact of simulation training in pediatric extracorporeal cardiopulmonary resuscitation (ECPR) and reveal targets for further training 
(42). ACTA is a streamlined method of CTA used to assess aspect of expertise and present findings in an operational manner (43). This method exposed two behaviors (coordination of compression with surgical cannulation and performance of sterile compressions) which were targeted for further simulation training (42).

CTA during the design or improvement of neonatal resuscitation simulations could follow any of the methods described above. CTA could be used to determine the content and structure of scenarios, develop these training scenarios, and develop performance metrics based on the scenarios developed.

\section{ASSESSMENT}

CTA-based assessments can be used to evaluate technical and non-technical skills in a clinical setting. Non-technical skills (i.e., communication, leadership, and situational awareness) are core competencies in clinical practice $(44,45)$. During neonatal resuscitations, these skills are particularly important as they have been identified as causes of human error (35). While no study has used CTA in the development of a non-technical assessment specific to neonatal resuscitation, these methods have been successfully used in other medical disciplines including surgery and anesthesiology (46-48). The Anesthetists' Non-Technical Skills assessment tool, for example, was developed using CTA and has been validated with high interrater reliability in both a clinical and simulated environment (49). Anesthetists' Non-Technical Skills assessment tool has since been used in neonatal resuscitation (50). Szulewski et al. performed a CTA of residents who completed simulationbased emergency room resuscitation exams. This approach allowed for a qualitative characterization of the cognitive processes underlying resident's crisis resource management and an examination of how these skills varied with resident's performance (51). This information could be applied to improve non-technical assessment of residents performing simulationbased resuscitation.

Similarly, CTA has been used to assess technical skills during simulations (52), scenario testing (53), and checklists (54), which are part of new surgical performance metrics. Recently, a surgical competency assessment tool to assess technical and non-technical components of transurethral resection of a bladder tumor was developed using CTA with reported feasibility, validity $(r>0.5$, $p<0.01$ ), and reliability (coefficient Phi $\geq 0.8$ ) (55). The method used to create these tools could be adapted to assess technical and non-technical skills during neonatal resuscitation.

\section{LIMITATIONS OF COGNITIVE TASK ANALYSIS}

There are several limitations to CTA methods. Most studies do not describe the CTA method used in sufficient detail to reproduce the study. CTA can be a tedious and time-consuming method to obtain information (56). Furthermore, the quality of information produced is determined by the level of SME; therefore, the variability in the definition of expertise might translate to variable CTA results. Finally, due to the large number of CTA methods available, it is difficult to identify the optimal CTA method for a given clinical context.

\section{APPLICATION OF CTA TO NEONATAL RESUSCITATION}

CTA could be applied to neonatal resuscitation. Specifically, CTA could be used to improve resuscitation equipment, design clinical DSTs, assess teamwork and communication, and inform the development of training and assessment tools. As neonatal resuscitation is largely guided by standardized algorithms, CTA could also be used to study causes of algorithm deviations, analyze how algorithm adherence differs between experts and novices, and identify when experts might decide to deviate from an algorithm.

CTA could also be used to study the integration of advanced monitoring equipment into neonatal resuscitation. To supplement electrocardiogram (ECG) and pulse oximetry $\left(\mathrm{SpO}_{2}\right)$ currently recommended by international neonatal resuscitation guidelines, additional monitoring including near cerebral infrared spectroscopy (NIRS) and respiratory function monitoring (RFM) are being investigated (57, 58). CTA could be used to examine how HCPs integrate information provided by these monitors into established clinical decision pathways. This knowledge could be used to design monitor displays to present information in a manner that best fits the pre-existing cognitive framework of HCPs, thus optimizing usability while minimizing additional cognitive demands. CTA could also be used to guide the design of a DST that integrates ECG, $\mathrm{SpO}_{2}$, NIRS, RFM with standard neonatal resuscitation algorithms.

Neonatal resuscitation presents unique challenges to the application of CTA methods. Given the fast-paced and demanding nature of neonatal resuscitation, it may not be feasible to use CTA methods that occur during actual resuscitations (e.g., Think Aloud), as this may increase cognitive burden and compromise clinical performance. Instead, CTA methods that must be conducted during task completion could be performed during simulated neonatal resuscitation. Alternatively, videos recordings of resuscitations could be used to prompt recall. Mobile eye-tracking glasses have been used to analyze visual attention of HCPs during neonatal resuscitation (59). These glasses use reflected infrared light to track pupillary movements while simultaneously recording video from the wearer's viewpoint. The resulting video indicates the wearer's visual focus during the performance of clinical tasks (e.g., what they were looking at when performing bag-mask ventilation). Thus, eye-tracking recordings can be used to provide an information-rich prompt for the recall of knowledge, cognitive tasks, and decision-making after an actual neonatal resuscitation event. Recently, Katz et al. used the Think Aloud method along with mobile eye-tracking glasses to analyze how neonatal HCPs 
interact with respiratory function monitors during simulated positive pressure ventilation. This technique allowed the authors to confirm that HCPs cognitive attention corresponded with their visual attention (60).

Given the diverse physical and social environments in which neonatal resuscitation occur and the variety of HCP roles required to complete this task, it is unlikely that the cognitive pathways exposed using CTA in one context will be generalizable to all settings. Knowledge elicited from an HCP practicing in a tertiary care center may not be generalizable to an HCP practicing in a low-resource setting. Similarly, SME from different disciplines (physician, nursing, respiratory therapy, midwifery, etc.) may offer significantly different perspectives despite applying the same CTA methods in the same clinical context. CTA that examines neonatal resuscitation across settings and roles is therefore needed to accurately inform improvements to neonatal resuscitation equipment, DST, teamwork, and training.

\section{CONCLUSIONS}

CTA is a method to elicit expertise, which then can be applied to improve medical technology, clinical DSTs, teamwork, and training. The application of CTA to neonatal resuscitation has

\section{REFERENCES}

1. Cutumisu M, Brown MRG, Fray C, Schmölzer GM. Growth mindset moderates the effect of the neonatal resuscitation program on performance in a computer-based game training simulation. Front Pediatr. (2018) 6:195. doi: 10.3389/fped.2018.00195

2. Patel J, Posencheg M, Ades A. Proficiency and retention of neonatal resuscitation skills by pediatric residents. Pediatrics. (2012) 130:515-21. doi: 10.1542/peds.2012-0149

3. Yamada NK, Halamek LP. On the need for precise, concise communication during resuscitation: a proposed solution. J Pediatr. (2015) 166:184-7. doi: 10.1016/j.jpeds.2014.09.027

4. Thomas EJ, Sexton JB, Lasky RE, Helmreich RL, Crandell DS, Tyson J. Teamwork and quality during neonatal care in the delivery room. J Perinatol. (2006) 26:163-9. doi: 10.1038/sj.jp.7211451

5. The Joint Commission. Sentinel Event Alert: Issue 30 Preventing Infant Death and Injury During Delivery. (2004). Available online at: https://www. jointcommission.org/sentinel_event_alert_issue_30_preventing_infant_ death_and_injury_during_delivery_additional_resources (accessed January 15, 2019).

6. Schraagen JM, Chipman SF, Shalin VL. Cognitive Task Analysis. Mahwah, NJ: Lawrence Erlbaum Associates (2000).

7. Clark R, Feldon D, van Merrienboer JJ, Yates K, Early S. Cognitive task analysis. In: Handbook of Research on Educational Communications and Technology. (2008). Available online at: https://digitalcommons.usu.edu/itls_ facpub/366 (accessed December 20, 2018).

8. Cooke NJ. Varieties of knowledge elicitation techniques. Int J Hum Comput Stud. (1994) 41:801-49. doi: 10.1006/ijhc.1994.1083

9. van Someren M, Yvonne B, Sandberg J. The Think Aloud Method: A Practical Approach to Modelling Cognitive. London: Academic Press (1994).

10. Klein GA, Calderwood R, MacGregor D. Critical decision method for eliciting knowledge. IEEE Trans Syst Man Cybern. (1989) 19:462-72. doi: $10.1109 / 21.31053$

11. Li $\mathrm{H}, \mathrm{Ku} \mathrm{M}$, Schumacher R, Seagull FJ. Designing automated aids for patient monitoring systems in intensive care units. Proc Int Symp the potential to improve knowledge in novice learners, reduce human errors, and improve outcomes for newborn infants.

\section{AUTHOR CONTRIBUTIONS}

GS, BL, and EZ: conception and design, collection and assembly of data, analysis and interpretation of the data, drafting of the article, critical revision of the article for important intellectual content, and final approval of the article.

\section{ACKNOWLEDGMENTS}

We would like to thank the public for donating money to our funding agencies: EZ was supported by the Maternal and Child Health Scholarship Program (MatCH), Faculty of Medicine and Dentistry, University of Alberta. BL was supported by the Stollery Clinical Research Fellowship, University of Alberta. GS is a recipient of the Heart and Stroke Foundation/University of Alberta Professorship of Neonatal Resuscitation, a National New Investigator of the Heart and Stroke Foundation Canada and an Alberta New Investigator of the Heart and Stroke Foundation Alberta. This research was supported by the Women and Children's Health Research Institute grant through the generous support of the Stollery Children's Hospital Foundation.

Hum Factors Ergon Heal Care. (2013) 2:69-76. doi: 10.1177/23278579130 21014

12. Ferris TK, Shepley MM. The design of neonatal incubators: a systemsoriented, human-centered approach. J Perinatol. (2013) 33:S24-31. doi: $10.1038 /$ jp.2013.11

13. Kawamoto $\mathrm{K}$, Houlihan $\mathrm{CA}$, Balas $\mathrm{EA}$, Lobach DF. Improving clinical practice using clinical decision support systems: a systematic review of trials to identify features critical to success. BMJ. (2005) 330:765. doi: $10.1136 /$ bmj.38398.500764.8F

14. Leppink J, van den Heuvel A. The evolution of cognitive load theory and its application to medical education. Perspect Med Educ. (2015) 4:119-27. doi: 10.1007/s40037-015-0192-x

15. Fuerch JH, Yamada NK, Coelho PR, Lee HC, Halamek LP. Impact of a novel decision support tool on adherence to Neonatal Resuscitation Program algorithm. Resuscitation. (2015) 88:52-6. doi: 10.1016/j.resuscitation.2014.12.016

16. Schnittker R, Marshall S, Horberry T, Young K, Lintern G. Examination of anesthetic practitioners' decisions for the design of a cognitive tool for airway management. Proc Hum Factors Ergon Soc Annu Meet. (2016) 60:1765-9. doi: $10.1177 / 1541931213601404$

17. Schnittker R, Marshall S, Horberry T, Young KL. The co-design process of a decision support tool for airway management. In: Proceedings of the 20th Congress of the International Ergonomics Association (IEA). Springer Nature (2018). p. 111-20.

18. Baxter GD, Monk AF, Tan K, Dear PRF, Newell SJ. Using cognitive task analysis to facilitate the integration of decision support systems into the neonatal intensive care unit. Artif Intell Med. (2005) 35:243-57. doi: 10.1016/j.artmed.2005.01.004

19. Shibl R, Lawley M, Debuse J. Factors influencing decision support system acceptance. Decis Support Syst. (2013) 54:953-61. doi: 10.1016/j.dss.2012.09.018

20. Sheehan B, Kaufman D, Bakken S, Currie LM. Cognitive analysis of decision support for antibiotic ordering in a neonatal intensive care unit. Appl Clin Inform. (2012) 3:105-23. doi: 10.4338/ACI-2011-10RA-0060 
21. Polson P, Lewis C, Rieman J, Wharton C. Cognitive walkthroughs: a method for theory-based evaluation of user interfaces. Int J Man Mach Stud. (1992) 36:741-73. doi: 10.1016/0020-7373(92)90039-N

22. Manser T. Teamwork and patient safety in dynamic domains of healthcare: a review of the literature. Acta Anaesthesiol Scand. (2009) 53:143-51. doi: 10.1111/j.1399-6576.2008.01717.x

23. McHugh A, Crandall B, Miller T. Barriers and facilitators of common ground in critical care teams. Proc Hum Factors Ergon Soc Annu Meet. (2006) 50:9348. doi: 10.1177/154193120605001029

24. Custer JW, White E, Fackler JC, Xiao Y, Tien A, Lehmann H, et al. A qualitative study of expert and team cognition on complex patients in the pediatric intensive care unit. Pediatr Crit Care Med. (2012) 13:278-84. doi: 10.1097/PCC.0b013e31822f1766

25. Schraagen JM, Schouten T, Smit M, Haas F, van der Beek D, van de Ven J, et al. Assessing and improving teamwork in cardiac surgery. Qual Saf Health Care. (2010) 19:e29. doi: 10.1136/qshc.2009.040105

26. Sadideen H, Wilson D, Moiemen N, Kneebone R. Proposing "The Burns Suite" as a novel simulation tool for advancing the delivery of burns education. $J$ Burn Care Res. (2014) 35:62-71. doi: 10.1097/BCR.0b013e31829b371d

27. Elen J, Clark RE, European Association for Research on Learning and Instruction. When less is more: research and theory insights about instruction for complex learning. In: Handling Complexity in Learning Environments: Theory and Research. 1st ed. Oxford, UK; Boston, MA: Elsevier (2006). p. 311.

28. Sullivan ME, Yates KA, Inaba K, Lam L, Clark RE. The use of cognitive task analysis to reveal the instructional limitations of experts in the teaching of procedural skills. Acad Med. (2014) 89:811-6. doi: 10.1097/ACM.0000000000000224

29. Clark R. Cognitive task analysis for expert-based instruction in healthcare. In: Spector J, Merrill M, Elen J, Bishop M, editors. Handbook of Research on Educational Communications and Technology. New York, NY: Springer (2014). p. 541-51. doi: 10.1007/978-1-4614-3185-5_42

30. Tofel-Grehl C, Feldon DF. Cognitive task analysis-based training. J Cogn Eng Decis Mak. (2013) 7:293-304. doi: 10.1177/1555343412 474821

31. Wingfield LR, Kulendran M, Chow A, Nehme J, Purkayastha S. Cognitive task analysis bringing olympic athlete style training to surgical education. Surg Innov. (2015) 22:406-17. doi: 10.1177/1553350614556364

32. Crandall B, Getchell-Reiter K. Critical decision method: a technique for eliciting concrete assessment indicators from the intuition of NICU nurses. Adv Nurs Sci. (1993) 16:42-51. doi: 10.1097/00012272-19930900000006

33. Militello L, Nursing L. Patient assessment skills: assessing early cues of necrotizing enterocolitis. J Perinat Neonatal Nurs. (1995) 9:42-52. doi: 10.1097/00005237-199509000-00007

34. Patterson MD, Militello LG, Bunger A, Taylor RG, Wheeler DS, Klein G, et al. Leveraging the critical decision method to develop simulation-based training for early recognition of sepsis. J Cogn Eng Decis Mak. (2016) 10:36-56. doi: $10.1177 / 1555343416629520$

35. Mileder LP, Urlesberger B, Szyld EG, Roehr CC, Schmölzer GM. Simulation-based neonatal and infant resuscitation teaching: a systematic review of randomized controlled trials. Klin Padiatr. (2014) 226:259-67. doi: 10.1055/s-0034-1372621

36. Cusack J, Fawke J. Neonatal resuscitation: are your trainees performing as you think they are? A retrospective review of a structured resuscitation assessment for neonatal medical trainees over an 8-year period. Arch Dis Child Fetal Neonatal Ed. (2012) 97:F246-8. doi: 10.1136/archdischild-2011-300548

37. Cannon-Bowers J, Bowers C, Stout R, Ricci K, Hildabrand A. Using cognitive task analysis to develop simulation-based training for medical tasks. Mil Med. (2013) 178:15-21. doi: 10.7205/MILMED-D-13-00211

38. Masiello I. Why simulation-based team training has not been used effectively and what can be done about it. Adv Heal Sci Educ. (2012) 17:279-88. doi: 10.1007/s10459-011-9281-8

39. Spector JM, Merrill MD, van Merrienboer J, Driscoll MP. Handbook of Research on Educational Communications and Technology: A Project of the Association for Educational Communications and Technology. 3rd ed. New York, NY: Taylor \& Francis (2007).

40. Pfandler M, Stefan P, Wucherer P, Lazarovici M, Weigl M. Stepwise development of a simulation environment for operating room teams: the example of vertebroplasty. Adv Simul. (2018) 3:18. doi: 10.1186/s41077-018-0077-2

41. Nimmo GR, Wylie G, Scarth J, Simpson J, Gracie E, Torrance I, et al. Critical events simulation for neonatal and paediatric extracorporeal membrane oxygenation. J Intens Care Soc. (2008) 9:20-2. doi: $10.1177 / 175114370800900107$

42. Sawyer T, Burke C, McMullan DM, Chan T, Valdivia H, Yalon L, et al. Impacts of a pediatric extracorporeal cardiopulmonary resuscitation (ECPR) simulation training program. Acad Pediatr. (2019) 19:566-71. doi: 10.1016/j.acap.2019.01.005

43. Militello LG, Hutton RJB. Applied cognitive task analysis (ACTA): a practitioner's toolkit for understanding cognitive task demands. Ergonomics. (1998) 41:1618-41. doi: 10.1080/001401398186108

44. Flin R, Yule S, Paterson-Brown S, Maran N, Rowley D, Youngson G. Teaching surgeons about non-technical skills. Surg. (2007) 5:86-9. doi: 10.1016/S1479-666X(07)80059-X

45. Buist M, Harrison J, Abaloz E, Van Dyke S. Six year audit of cardiac arrests and medical emergency team calls in an Australian outer metropolitan teaching hospital. BMJ. (2007) 335:1210-2. doi: 10.1136/bmj.39385.53 4236.47

46. Fletcher G, Flin R, McGeorge P, Glavin R, Maran N, Patey R. Rating non-technical skills: developing a behavioural marker system for use in anaesthesia. Cogn Technol Work. (2004) 6:165-71. doi: 10.1007/s10111-0040158-y

47. Flin R, Fletcher G, McGeorge P, Glavin R, Maran N, Patey R. Rating anaesthetists' non-technical skills-the Ants System. Proc Hum Factors Ergon Soc Annu Meet. (2003) 47:1498-501. doi: 10.1177/154193120304701227

48. Yule S, Flin R, Paterson-Brown S, Maran N, Rowley D. Development of a rating system for surgeons' non-technical skills. Med Educ. (2006) 40:1098104. doi: 10.1111/j.1365-2929.2006.02610.x

49. Boet S, Larrigan S, Martin L, Liu H, Sullivan KJ, Etherington N. Measuring non-technical skills of anaesthesiologists in the operating room: a systematic review of assessment tools and their measurement properties. Br J Anaesth. (2018) 121:1218-26. doi: 10.1016/j.bja.2018.07.028

50. Cavicchiolo ME, Cavallin F, Staffler A, Pizzol D, Matediana E, Wingi $\mathrm{OM}$, et al. Decision making and situational awareness in neonatal resuscitation in low resource settings. Resuscitation. (2019) 134:41-8. doi: 10.1016/j.resuscitation.2018.10.034

51. Szulewski A, Braund H, Egan R, Gegenfurtner A, Hall AK, Howes D, et al. Starting to think like an expert: an analysis of resident cognitive processes during simulation-based resuscitation examinations. Ann Emerg Med. (2019). doi: 10.1016/j.annemergmed.2019.04.002. [Epub ahead of print].

52. D'Angelo A-LD, Rutherford DN, Ray RD, Laufer S, Kwan C, Cohen $\mathrm{ER}$, et al. Idle time: an underdeveloped performance metric for assessing surgical skill. Am J Surg. (2015) 209:645-51. doi: 10.1016/j.amjsurg.2014. 12.013

53. Kyaw Tun J, Granados A, Mavroveli S, Nuttall S, Kadiyala AN, Brown $\mathrm{R}$, et al. Simulating various levels of clinical challenge in the assessment of clinical procedure competence. Ann Emerg Med. (2012) 60:112-20.e5. doi: 10.1016/j.annemergmed.2012.01.036

54. D’Angelo A-LD, Cohen ER, Kwan C, Laufer S, Greenberg C, Greenberg J, et al. Use of decision-based simulations to assess resident readiness for operative independence. Am J Surg. (2015) 209:132-9. doi: 10.1016/j.amjsurg.2014.10.002

55. de Vries AH, Muijtjens AMM, van Genugten HGJ, Hendrikx AJM, Koldewijn EL, Schout BMA, et al. Development and validation of the TOCO-TURBT tool: a summative assessment tool that measures surgical competency in transurethral resection of bladder tumour. Surg Endosc. (2018) 32:4923-31. doi: 10.1007/s00464-018-6251-8

56. Riggle JD, Wadman MC, McCrory B, Lowndes BR, Heald EA, Carstens $\mathrm{PK}$, et al. Task analysis method for procedural training curriculum development. Perspect Med Educ. (2014) 3:204-18. doi: 10.1007/s40037-0130100-1

57. Pichler G, Baumgartner S, Biermayr M, Dempsey E, Fuchs H, Goos TG, et al. Cerebral regional tissue Oxygen Saturation to Guide Oxygen Delivery in preterm neonates during immediate transition after birth (COSGOD III): an investigator-initiated, randomized, multi-center, multi-national, clinical trial on additional cerebral tissue oxygen saturation monitoring 
combined with defined treatment guidelines versus standard monitoring and treatment as usual in premature infants during immediate transition: study protocol for a randomized controlled trial. Trials. (2019) 20:178. doi: 10.1186/s13063-019-3258-y

58. Schmölzer GM, Morley CJ, Wong C, Dawson JA, Kamlin CO, Donath SM, et al. Respiratory function monitor guidance of mask ventilation in the delivery room: a feasibility study. J Pediatr. (2012) 160:377-81.e2. doi: 10.1016/j.jpeds.2011.09.017

59. Law BHY, Cheung P-Y, Wagner M, van Os S, Zheng B, Schmölzer G. Analysis of neonatal resuscitation using eye tracking: a pilot study. Arch Dis Child Fetal Neonatal Ed. (2018) 103:F82-4. doi: 10.1136/archdischild-2017313114

60. Katz TA, Weinberg DD, Fishman CE, Nadkarni V, Tremoulet P, Te Pas AB, et al. Visual attention on a respiratory function monitor during simulated neonatal resuscitation: an eye-tracking study. Arch Dis Child Fetal Neonatal Ed. (2019) 104:F259-64. doi: 10.1136/archdischild-2017-314449

Conflict of Interest Statement: The authors declare that the research was conducted in the absence of any commercial or financial relationships that could be construed as a potential conflict of interest.

Copyright (๑) 2019 Zehnder, Law and Schmölzer. This is an open-access article distributed under the terms of the Creative Commons Attribution License (CC BY). The use, distribution or reproduction in other forums is permitted, provided the original author(s) and the copyright owner(s) are credited and that the original publication in this journal is cited, in accordance with accepted academic practice. No use, distribution or reproduction is permitted which does not comply with these terms. 